\title{
INERT TRACER GAS WASHOUT FROM MIXED VENOUS BLOOD: THE SLOPING ALVEOLAR PLATEAL
}

\author{
W. R. DE VRIES', S.C. M. LUIJENDIJK ${ }^{1}$ and A. ZWART ${ }^{2}$ \\ 'Department of Physiology, State University Utrecht and Institute of Medical Physics T.N.O.. Litrecht, \\ The Netherlands
}

\begin{abstract}
The aim of this model study was to investigate the mechanisms underlying the sloping alveolar plateau for inert tracer gases supplied to the lung by mixed venous blood. Transpulmonary gas exchange was simulated in an asymmetric lung model for conditions at rest and in exercise. For highly soluble gases, the calculations show that the varying amount of tracer gas dissolved in superficial parenchymal tissuc and capillary blood causes a sustained stratification in the acinus during expiration and that this is mainly responsible for the slope. For this type of tracer gas, the slope is almost independent of variations in the molecular diffusion coefficient (D) of the gases. In contrast, for poorly soluble gases, the contributions of local parallel inhomogeneities of gas concentrations in the acinus and the continued gas exchange across the alveolo-capillary membrane are mainly responsible for the slope. The first factor, which depends on the asymmetric branching pattern of intra-acinar airways, increases with decreasing $D$ values. The contribution of continued gas exchange to the slope is most pronounced under exercise conditions. This contribution is almost independent of the bloodigas partition coefficient, $i$, for $i$ values less than 4.0 .
\end{abstract}

Asymmetric lung model

Blood'gas partition coefficient

Molecular diffusion coefficient
Synchronous ventilation

Transpulmonary gas exchange

In previous model calculations (Luijendijk et al., 1980: de Vries et al., 1981) dealing with the washout of helium and sulfur hexafluoride from the alveolar space, we observed that the asymmetry of the branching pattern of the intra-acinar airways gives rise to parallel concentration inhomogeneities at a local level. This finding is in agreement with results of others using asymmetric lung models (Mon and Ultman, 1976; Paiva and Engel, 1979; Davidson and Engel, 1982). These inhomogeneities are, among others, responsible for the sloping alveolar plateau and its properties (Luijendijk et al., 1980; de Vries et al., 1981; Paiva and Engel, 1981). This places the question of the origin of the slope for healthy subjects in a new perspective. 
In this paper, we focus on the mechanisms underlying the sloping alveolar plateau for tracer gases supplied to the lung by circulating blood. For this purpose, we simulate the multiple inert gas elimination method described by Wagner et al. (1974). The results of the calculations concern the slope of the alveolar platea as a function of the blood/gas partition coefficient, $\lambda$, and the molecular diffusion coefficient, $\mathrm{D}$, for different ventilation-perfusion conditions ranging from at rest to exercise. These model calculations increase our understanding of the relative contributions of various determinants to the slope, such as the intra-acinar parallel concentration inhomogeneities, the continued gas exchange across the alveolocapillary membrane and the effect of tidal variations in the amount of dissolved tracer gas in superficial parenchymal tissue and capillary blood, which was not previously considered as making an important contribution to the slope.

\section{Lung model}

In previous work (Luijendijk et al., 1980; de Vries et al., 1981), we developed a lung model for studying intrapulmonary gas mixing for poorly soluble tracer gases. The geometry of that model was based on morphometric data published by Weibel (1963), Hansen et al. (1975) and Hansen and Ampaya (1975). Its dimensions are almost the same as those given by Lacquet (1976). For a detailed description of the lung model, the reader is referred to previous publications (Luijendijk et al., 1980; de Vries et al., 1981; de Vries and Luijendijk, 1982). Only the basic properties will be summarized here. The model consists of an inelastic and an elastic part. The inelastic part represents the larger airways from the mouth up to and including the 10th gencration airways. It constitutes a fixed lumenal volume representing the anatomical dead space volume where only convective gas mixing takes place during breathing. The elastic part (11.26th generation airways) represents the region where intrapulmonary gas mixing and gas exchange between the gas phase and the dissolved phase takes place. The dimensions of the elastic part (lumenal volume. cross-sectional area and length) vary during a breathing cycle, accounting for the oscillatory nature of breathing. The branching pattern of airways in the clastic part is asymmetric. Based on a dichotomous branching pattern, the asymmetry starts at a particular generation designated as the generation of division $(\mathrm{N})$. Each airway of the $(\mathrm{N}-1)$ th generation forms the parent branch of a single pair of parallel lung units. In one lung unit, the branching pattern is reduced by cutting off a certain number of generations. All airways of this reduced unit end at the 21 st gencration. All airways of the other nonreduced unit end at generation number 26 (see fig. 1). The dimensions of airways in corresponding generations in the reduced and nonreduced units are equal. Different choices for the start as well as the extent of branching pattern asymmetry will result in a large number of asymmetric lung models. Therefore, we limit these choices to a few relevant examples indicated by the code $\mathrm{N} ; 26-21 ; 1$, where $\mathrm{N}$ is the number of the generation of division, ranging 


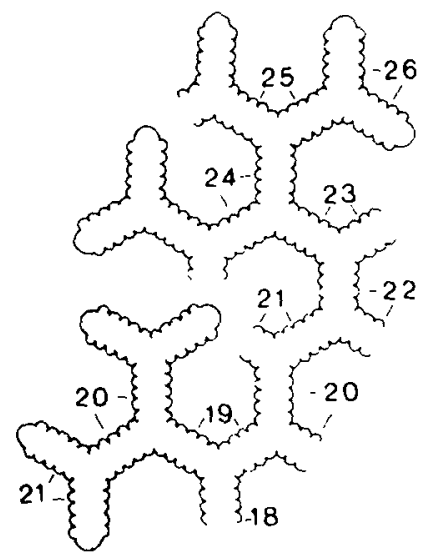

Fig. 1. The asymmetry of the intra-acinar branching pattern. After generation 18 one of the two branches of generation 19 continues to generation 21 (reduced unit, shaded area). The other branch supplies a nonreduced parallel unit which ends at generation 26 . The ratio of the lumenal volume of the reduced and nonreduced unit is about $1: 5$. In this cxample, $N=19$.

from 16 up to 21 : index ' $26-21$ ' indicates the degree of asymmetry and index ' 1 ' is the index for local tidal volume to local volume distribution. In fact, it represents the value of $\Delta \mathrm{V} / \mathrm{V}$ in the reduced units divided by $\Delta \mathrm{V} / \mathrm{V}$ in the nonreduced units. Such a local $\Delta V / V$ distribution is not an essential restriction, since de Vries et al. (1981) showed that local $\Delta \mathrm{V} / \mathrm{V}$ inhomogeneities in such a model hardly influence the slope.

The model is extended with pulmonary perfusion. Q.p, to enable the simulation of washout experiments for tracer gases supplied to the lung by mixed venous blood. A homogeneous $\dot{\mathrm{Q}} \mathrm{p} / \mathrm{V}$ distribution is assumed, indicating a homogeneous $\dot{\mathrm{V}} / \dot{\mathrm{Q}} \mathrm{p}$ distribution throughout the elastic part. Shunt perfusion is omitted in the calculations. To account for the effect of tidal variations in the amount of dissolved tracer gas in parenchymal tissue and capillary blood on the gas exchange, the lung model is further extended by adding a virtual volume to its gas volume. According to calculations of Zwart et al. (1982), the superficial parenchymal tissue in the inelastic part can be neglected, as the effective tissue volume is very small in comparison with that of the elastic part. In addition, it has to be considered that the surface-tovolume ratio and the resident time of gas in the upper airways are much less than in the distal airways. Therefore, the interaction of gas molecules with the walls of upper airways will be much smaller than with the walls of distal airways. As a consequence, the virtual volume is divided only among the elastic part in proportion to the lumenal volumes of airway generations. For most tracer gases applied in the multiple inert gas elimination technique, the water/gas, blood/gas and tissue/gas partition coefficients appear to be about equal (Cander, 1959; Wagner et al., 1974). Therefore, we assume equal blood/gas and tissuc/gas partition coefficients. In that case, the virtual volume equals the product of the blood/gas partition coefficient and the total volume of the alveolo-capillary membrane and capillary blood. According to Weibel (1963) the latter total volume is about $200 \mathrm{ml}$. 
For the analysis of the relative contributions of various determinants to the slope. two modifications of this extended lung model are used. namely, a tissuc-free model and a perfusion-interrupted model. In the tissuc-free model. the virtual volume is omitted. The comparison between the results of calculations with and without virtual volume gives the opportunity to analyse the specific contribution of tidal variations in the amount of dissolved tracer gas in tissue and capillary blood to the slope. The perfusion interrupted model simulates a situation where the pulmonary artery is occluded for a single expiration. from which the slope is calculated. The comparison between this slope and that of the previous expiration with perfusion enables us to analyse the specific contribution of continued gas exchange across the alveolo-capillary membranc to the slope.

\section{Numerical procedure and material}

To enable the use of difference equations, the lung model is subdivided into 341 serial compartments. The inelastic part $(0-10$ th generation) contains 2.35 compartments having an equal volume of $0.97 \mathrm{ml}$. The elastic part $(11-26 \mathrm{th}$ generation $)$ contains the remaining 106 compartments. Before cach calculation, the dimensions of the model are scaled up to a preselccted total lung capacity (TLC) of $6.5 \mathrm{~L}$.

The convective gas mixing in the inclastic part is accounted for by using a mixing factor of 0.25 for all tracer gases. This factor describes the fraction of compartmental gas that is exchanged with each of the two adjacent compartments during each time interval that $0.97 \mathrm{ml}$ is inspired or expired. After such a time interval. the compartmental concentrations are updated and the adjusted concentration profile obtained in this way is then moved one compartment further in the direction of the flow. This procedure results in an axial dispersion that can be described by a Gaussian function (Ultman and Thomas, 1979), which is fully characterized by its width at half height. The mixing factor of 0.25 is equivalent to a width at half height of $25 \mathrm{ml}$. This selection of the mixing factor is based on unpublished data on axial dispersion in a glass tube model of upper airway generations.

The local gas transport in the elastic part of the model is calculated by means of two separate equations. The mass transport across the compartmental part of the alveolo-capillary membrane during the time interval $(t, t+\Delta t)$ is given by:

$$
n_{1}=\dot{q} \cdot(C \dot{v}-\dot{i} C) \cdot \Delta t
$$

and the mass transport by diffusion and convection in the gas phase between successive compartments is given by:

$$
\mathrm{n}_{2}=-\mathrm{D} \cdot \mathrm{S} \cdot(\Delta \mathrm{C} / \Delta \mathrm{l}) \cdot \Delta \mathrm{t}+\dot{\mathrm{V}} \cdot \mathrm{C} \cdot \Delta \mathrm{t}
$$

where:

$\mathrm{n}_{1}, \mathrm{n}_{2}=$ mass transport during the time interval $(t . t+\Delta \mathrm{t})$

$\mathrm{D}=$ molecular diffusion coefficient 
$\mathrm{S} \quad=$ cross sectional area of the imaginary dividing surfacc between successive compartments

$\Delta \mathrm{C}=$ concentration difference between successive compartments

$\Delta \mathrm{l} \quad=$ axial length of a compartment

$\dot{\mathrm{V}}=$ local flow of the gas mixture

$\mathrm{C}=$ local concentration of tracer gas in the gas phase

$\dot{\mathrm{q}}=$ compartmental perfusion

$\mathrm{C} \overline{\mathrm{V}}=$ concentration of tracer gas in mixed venous blood

$\lambda \quad=$ blood/gas partition coefficiont of tracer gas

From eq. 1, one obtains $n_{1}$ as the mass transport from pulmonary blood to the gas phase in the compartment. From eq. 2, one obtains the mass transport between two successive compartments. In general, there are two adjacent compartments; therefore, eq. 2 must be applied twice. The mass transport across the two dividing surfaces of each compartment is denoted as $n_{2}$ and $n_{i}$, respectively. The new concentration in each compartment at time $t+\Delta t$ is then calculated from:

$$
\mathrm{C}(\mathrm{t}+\Delta \mathrm{t})=\left(\mathrm{V}(\mathrm{t}) \cdot \mathrm{C}(\mathrm{t})+\mathrm{n}_{1}+\mathrm{n}_{2}+\mathrm{n}_{3}\right) / \mathrm{V}(\mathrm{t}+\Delta \mathrm{t})
$$

where $V(t)$ and $V(t+\Delta t)$ are the comparimental volumes (gas volume + virtual volume) at time $t$ and $t+\Delta t$, respectively.

Additional assumptions and features of the calculations are:

- instantaneous radial concentration equilibrium within each compartment (Chang et al., 1973; Mon and Ultman, 1976);

- instantaneous partial pressure equilibrium between end capillary blood and the gas phase in the compartments;

- discrete time intervals $\Delta t(\mathrm{sec})$ less than $10^{-5} \mathrm{~cm}^{2} /$ molecular diffusion coefficient to obtain stable solutions;

- adjustment of the dimensions of the elastic compartments corresponding to the actual lung volume after each time interval $\Delta t$;

- synchronous inflation and deflation of the model;

- oscillatory breathing and nonpulsatile perfusion;

-- tissue $/$ gas partition coefficient $=$ blood $/$ gas partition coefficient .

- the computational procedure guarantees the conservation of mass.

The slopes of the alveolar plateaus are calculated from the end-expiratory part of the concentration curve if breath-to-breath steady-state conditions of gas exchange are attained. The slopes are expressed in percent per second and are calculated at between $90 \%$ and $100 \%$ of the expired volume.

The following ventilation-perfusion conditions are used:

I $\quad \dot{V} E=7.5 \mathrm{~L} / \mathrm{min} ; \quad f=15 / \mathrm{min} ; \quad \dot{Q p}=7.5 \mathrm{~L} / \mathrm{min}$

II $\dot{V} \mathrm{E}:=20.0 \mathrm{~L} / \mathrm{min} ; \quad f=20 / \mathrm{min} ; \quad \dot{\mathrm{p}}=10.0 \mathrm{~L} / \mathrm{min}$

III $\dot{V} E=60.0 \mathrm{~L} / \mathrm{min} ; \mathrm{f}=30 / \mathrm{min} ; \quad \dot{Q} p=20.0 \mathrm{~L} / \mathrm{min}$

where $\dot{V}_{F}$ is the expired volume per minute, $f$ is the respiratory frequency and $\dot{Q}_{p}$ is the pulmonary perfusion per minute. Each inspiration starts from functional residual capacity FRC $(50 \%$, TLC.); the inspiratory and expiratory flow rates are 
taken to be equal and constant, with an instantaneous reversal of llow between the two phases.

The following values for $i$ and $\mathrm{D}$ (in $\mathrm{cm}^{2} ; \mathrm{s}$ ) are selected:

$\hat{\lambda}=0.01 ; 0.2 ; 0.4 ; 0.7 ; 1.0 ; 1.5 ; 2.5 ; 5.0 ; 10.0 ; 30.0 ; 110.0$ and 330.0

$\mathrm{D}=0.1 ; 0.22 ; 0.5$ and, in addition for condition $11 \mathrm{I}, \mathrm{D}=0.01$.

All values correspond to hypothetical tracer gases covering the range of tracer gases normally used in experiments. Poorly and highly soluble gases are represented by the $\lambda$ values of 0.01 (helium, sulfur hexafluoride) and 330.0 (acetone), respectively. Heavy and light gases are represented by the $D$ values of 0.1 (halothane, sulfur hexafluoride) and 0.5 (helium, hydrogen), respectively. Finally, $D=0.01$ is added to account for hyperbaric conditions.

\section{Results and Discussion}

THE: SLOPE OF THE ALVIOLAR PLATHAL AS A FUNCTION OF THE BLOODGGAS PARTITION COIFFICIENT (;)

The influence of the bloodigas partition coefficient, $\lambda$, on the slope of the alveolar plateau is calculated in the asymmetric lung model coded $N ; 26-21 ; 1$, with $N=16$, $17,18,19$ and 20 . The calculations are carried out with a fixed value for $D(0.22)$ approximating the $\mathrm{D}$ values for gases like $\mathrm{O}_{2}$ and $\mathrm{CO}_{2}$. In the left hand part of table 1 , the results for different ventilation -perfusion conditions show that the slope depends on the choice of $N$, except for the largest values of $\lambda$. Further, for all values of $N$, the slope as a function of $\lambda$ shows the same trend for the same ventilation-perfusion condition. In the human lung, one may expect that an asymmetric branching pattern of airways exists at any level within the acinus and that it will vary from one acinus to another. Therefore. we have also calculated for each $\lambda$ value the mean value of the slopes obtained for the different $N$ values. In fig. 2, the curve indicated by the character $\nabla$ illustrates this mean value for the slopes as a function of $i$. It may represent a more realistic approximation of the in vivo situation, although an obvious limitation is still the inability to deal with the intluence which serially distributed generations of division have on each other (Davidson and Engel, 1982).

As far as quantitative aspects are concerned, only a small fraction of these results can be compared with experimental data. According to Ulmer et al. (1976). the slope of a capnogram for healthy subjects at rest is about $1.65 \mathrm{~mm} \mathrm{Hg} / \mathrm{sec}$, which is equivalent to about $4^{\circ} \mathrm{sec}$. During exercise $(200 \mathrm{~W}$, bicycle ergometer), this slope increases to $10 \mathrm{~mm} \mathrm{Hg} \mathrm{sec}$ or about $25^{\circ} \mathrm{sec}$. The effective blood gas partition cocfficient of $\mathrm{CO}_{2}$ is about 2.5 (Robertson and Hastala, 1977). Our calculated results for such a $i$ value $(4.49 \% \mathrm{sec}$ in condition $I$ and $20.36 \% \mathrm{sec}$ in exercise condition III; sce fig. 2) are in good agreement with the experimental results of Ulmer et al. (1976). 
The results of similar series of calculations with the perfusion-interrupted lung model are shown in the right hand part of table 1. The solid curve in fig. 2 represents

TABLE 1

The slope of the alveolar plateau (in percent per sec) as a function of $\lambda$ and $N$ for the lung model $N^{\prime} ; 26-21 ; 1$ and $D=0.22$. I, II and III represent the corresponding ventilation-perfusion conditions (see text). The right and left hand parts apply to the lung models with and without an interrupted perfusion, respectively

\begin{tabular}{|c|c|c|c|c|c|c|c|c|c|c|c|}
\hline \multirow[t]{2}{*}{$\lambda$} & \multicolumn{5}{|l|}{$\mathrm{N}$} & \multicolumn{5}{|l|}{$\mathrm{N}$} & \multirow[t]{2}{*}{ Condition } \\
\hline & 16 & 17 & 18 & 19 & 20 & 16 & 17 & 18 & 19 & 20 & \\
\hline 0.01 & 2.84 & 3.50 & 4.25 & 5.04 & 4.01 & 1.30 & 1.98 & 2.74 & 3.54 & 2.50 & 1 \\
\hline 0.2 & 2.85 & 3.52 & 4.26 & 5.05 & 4.08 & 1.33 & 2.00 & 2.76 & 3.58 & 2.60 & \\
\hline 0.4 & 2.87 & 3.53 & 4.27 & 5.06 & 4.15 & 1.36 & 2.02 & 2.78 & 3.62 & 2.70 & \\
\hline 0.7 & 2.92 & 3.57 & 4.29 & 5.08 & 4.25 & 1.43 & 2.08 & 2.83 & 3.69 & 2.86 & \\
\hline 1.0 & 2.99 & 3.62 & 4.32 & 5.11 & 4.36 & 1.52 & 2.14 & 2.89 & 3.77 & 3.03 & \\
\hline 1.5 & 3.11 & 3.73 & 4.39 & 5.18 & 4.56 & 1.69 & 2.29 & 3.01 & 3.92 & 3.32 & \\
\hline 2.5 & 3.43 & 4.02 & 4.62 & 5.39 & 5.00 & 2.14 & 2.69 & 3.36 & 4.29 & 3.96 & \\
\hline 5.0 & 4.70 & 5.22 & 5.68 & 6.34 & 6.38 & 3.88 & 4.34 & 4.85 & 5.72 & 5.91 & \\
\hline 10.0 & 8.97 & 9.39 & 9.63 & 10.01 & 10.39 & 9.71 & 10.08 & 10.32 & 10.86 & 11.47 & \\
\hline 30.0 & 33.28 & 33.46 & 33.44 & 33.42 & 33.45 & 45.40 & 45.57 & 45.51 & 45.48 & 45.54 & \\
\hline 110.0 & 51.96 & 51.95 & 51.95 & 51.95 & 51.95 & 80.14 & 80.10 & 80.10 & 80.10 & 80.10 & \\
\hline 330.0 & 45.71 & 45.71 & 45.71 & 45.71 & 45.71 & 70.48 & 70.48 & 70.48 & 70.48 & 70.48 & \\
\hline 0.01 & 6.83 & 8.15 & 10.33 & 10.84 & 7.51 & 0.38 & 1.86 & 4.26 & 4.85 & 1.24 & 11 \\
\hline 0.2 & 6.71 & 8.01 & 10.17 & 10.71 & 7.45 & 0.35 & 1.79 & 4.17 & 4.83 & 1.27 & \\
\hline 0.4 & 6.60 & 7.87 & 10.00 & 10.58 & 7.38 & 0.34 & 1.72 & 4.07 & 4.81 & 1.31 & \\
\hline 0.7 & 6.45 & 7.67 & 9.75 & 10.39 & 7.28 & 0.31 & 1.63 & 3.94 & 4.77 & 1.37 & \\
\hline 1.0 & 6.30 & 7.47 & 9.52 & 10.20 & 7.18 & 0.29 & 1.55 & 3.82 & 4.74 & 1.43 & \\
\hline 1.5 & 6.06 & 7.17 & 9.14 & 9.89 & 7.04 & 0.27 & 1.43 & 3.62 & 4.68 & 1.53 & \\
\hline 2.5 & 5.63 & 6.63 & 8.47 & 9.33 & 6.77 & 0.23 & 1.23 & 3.27 & 4.55 & 1.73 & \\
\hline 5.0 & 4.76 & 5.57 & 7.12 & 8.13 & 6.25 & 0.20 & 0.92 & 2.62 & 4.21 & 2.22 & \\
\hline 10.0 & 3.67 & 4.24 & 5.41 & 6.46 & 5.63 & 0.35 & 0.79 & 1.97 & 3.66 & 3.08 & \\
\hline 30.0 & 4.69 & 5.10 & 5.61 & 6.03 & 6.39 & 5.3 .3 & 5.73 & 6.13 & 6.69 & 7.60 & \\
\hline 110.0 & 24.96 & 24.95 & 25.01 & 24.99 & 24.99 & 46.11 & 45.97 & 45.96 & 45.91 & 45.90 & \\
\hline 330.0 & 32.93 & 32.92 & 32.92 & 32.92 & 32.92 & 65.29 & 65.27 & 65.27 & 65.27 & 65.27 & \\
\hline 0.01 & 21.66 & 22.17 & 25.46 & 30.56 & 24.81 & 0.03 & 0.74 & 5.01 & 11.62 & 4.80 & III \\
\hline 0.2 & 21.30 & 21.79 & 25.00 & 30.05 & 24.48 & 0.02 & 0.71 & 4.88 & 11.49 & 4.87 & \\
\hline 0.4 & 20.93 & 21.40 & 24.53 & 29.54 & 24.15 & 0.02 & 0.68 & 4.74 & 11.36 & 4.94 & \\
\hline 0.7 & 20.39 & 20.83 & 23.85 & 28.81 & 23,66 & 0.02 & 0.64 & 4.56 & 11.17 & 5.05 & \\
\hline 1.0 & 19.87 & 20.29 & 23.20 & 28.09 & 23.19 & 0.02 & 0.60 & 4.38 & 10.98 & 5.15 & \\
\hline 1.5 & 19.04 & 19.43 & 22.18 & 26.97 & 22.45 & 0.02 & 0.55 & 4.12 & 10.68 & 5.32 & \\
\hline 2.5 & 17.54 & 17.88 & 20.35 & 24.93 & 21.10 & 0.02 & 0.47 & 3.66 & 10.10 & 5.63 & \\
\hline 5.0 & 14.50 & 14.74 & 16.71 & 20.81 & 18.34 & 0.04 & 0.36 & 2.84 & 8.81 & 6.28 & \\
\hline 10.0 & 10.46 & 10.60 & 11.98 & 15.28 & 14.54 & 0.15 & 0.31 & 1.95 & 6.85 & 7.04 & \\
\hline 30.0 & 5.22 & 5.18 & 5.70 & 7.23 & 8.20 & 2.50 & 2.37 & 2.78 & 4.63 & 7.25 & \\
\hline 110.0 & 10.90 & 11.47 & 11.98 & 12.16 & 12.14 & 25.14 & 26.75 & 28.01 & 28.11 & 27.93 & \\
\hline 330.0 & 22.01 & 22.21 & 22.17 & 22.16 & 22.16 & 63.57 & 64.50 & 64.12 & 64.09 & 64.09 & \\
\hline
\end{tabular}



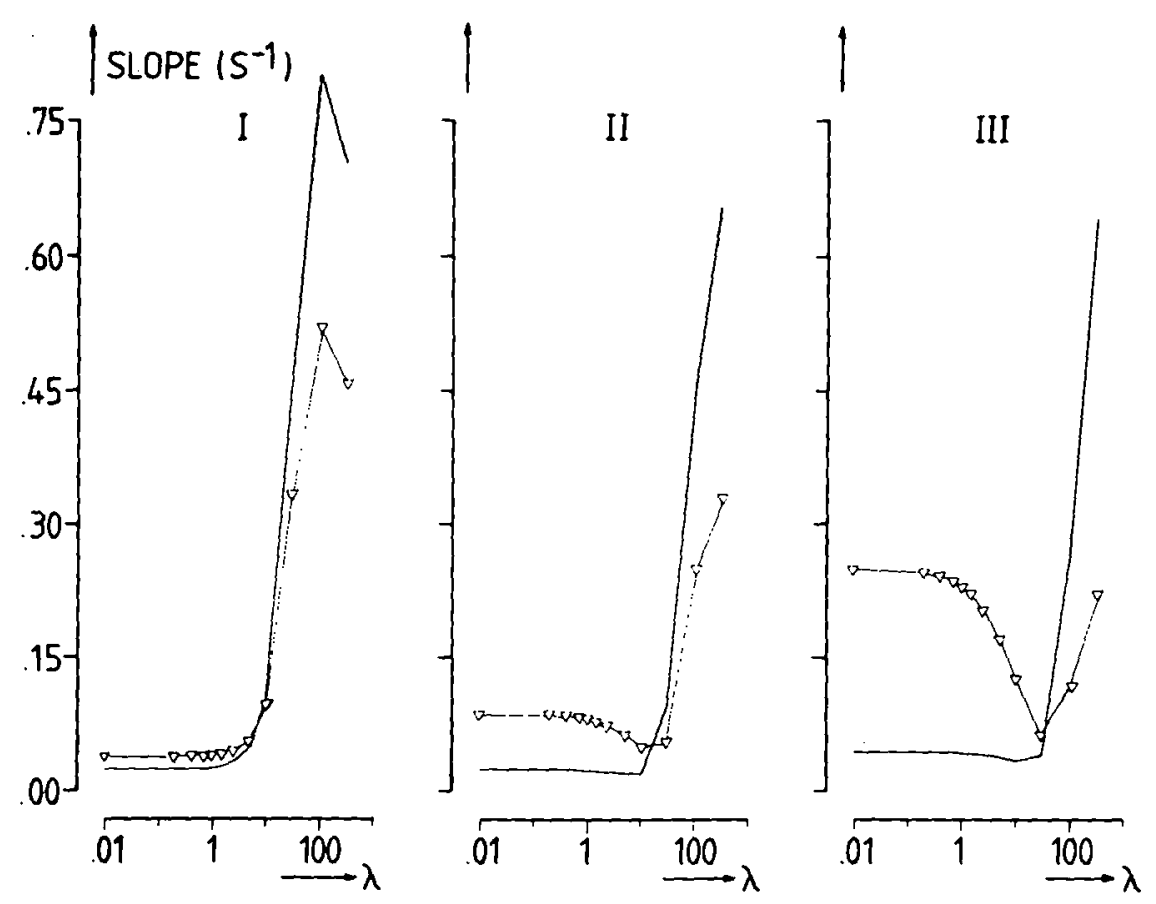

Fig. 2. The mean value for the slopes obtained for different $N$ values plotted as a function of $i$ for $\mathrm{D}=0.22$. Sections $\mathrm{I}$. II and III show the results for corresponding ventilation perfusion conditions (see text). The solid curve and the curve with triangles represent the lung model with and without interruption of perfusion, respectively.

the corresponding mean value for the slopes as a function of $\lambda$. The comparison between the results with and without perfusion interruption shows a positive contribution of continued gas exchange to the slope for $\lambda$ values less than about 10.0 . This positive contribution is nearly $\lambda$-independent for $\lambda<5.0$, but strongly depends on the ventilation-perfusion condition. In contrast, we observe a negative contribution of continued gas exchange to the slope for $\%$ values greater than about 10.0 and especially for $\lambda>110.0$. This negative contribution depends on both the ventilation-perfusion condition and the $\lambda$ values of the gases. How can these different observations for poorly and highly soluble gases be explained?

In the classical lung model with a nonoscillatory ventilation and perfusion, the alveolar compartment is taken to be homogeneous with a single value for the alveolar partial pressure of each tracer gas $(\mathrm{PA})$. However, an oscillatory ventilation is responsible for tidal fluctuations in $P_{A}$, resulting in a varying amount of dissolved tracer gas in pulmonary parenchymal tissue and capillary blood. This phenomenon plays an important role in transpulmonary gas exchange for highly soluble gases. which can be explained as follows.

Inspiration of tracer-free gas results in a transition zone betwcen inspired gas and residual gas in the gas-exchanging area. The surrounding lung tissue located 
proximally to this zone will release an amount of dissolved tracer gas into the adjacent airway lumen due to the low partial pressure, PA. This release results in a decrease in the partial pressure in lung tissue, $\mathrm{Pt}$, in such a way that the most proximally located tissue will have the lowest $\mathrm{Pt}$ because of the lowest pressure in the adjacent airway lumen. During expiration the transition zone moves rapidly outward and the resulting increase in partial pressure in the airway lumen induces a reversal of the partial pressure difference $\mathrm{Pt}-\mathrm{PA}$. Consequently, the tissue that releases most of the gas during inspiration also takes up most of the gas during expiration. In the case of the perfusion-interrupted model, the increase in $\mathrm{Pt}$ during expiration can only be caused by the supply of tracer-rich gas from distal airways. This increase in $\mathrm{Pt}$ proceeds for a longer period of time for highly soluble than for poorly soluble gases, because the buffering capacity of surrounding tissue is proportional to $\lambda$. Therefore, this phenomenon will be responsible for a sustained stratification in the airway lumen for highly soluble gases and this will contribute to the excessive increase in the slope for $\lambda$ values greater than about 10.0 (see results for the perfusion-interrupted model in table 1 and fig. 2). However, in the model with perfusion, the continued gas exchange across the alveolo-capillary membrane interacts with the consequences of the buffering capacity of surrounding tissue on the slope. In that case, the supply of tracer gas by perfusion accelerates the increase in $\mathrm{Pt}$ during expiration. This causes a more rapid decrease in stratification as compared with the perfusion-interrupted model. As a result, the continued gas exchange for highly soluble tracer gases reduces the slope of the alveolar plateau. This negative contribution will be most pronounced for condition III, where the supply by perfusion per unit lung volume is more than for conditions I and II (see fig. 2). Apparently, the presence of superficial lung tissue seems to be a necessary condition to generate a slope for highly soluble tracer gases. This is further emphasized in fig. 3, where the slopes for the normal lung model (curve with open circles) are compared with those of the tissue-free model (solid curve). For $\lambda$ values greater than 10.0 , the two curves deviate considerably from each other and, for $\lambda$ values greater than 100.0 , the slope of the tissue-free model even is zero.

For gases with a $\lambda$ value less than about 10.0 the buffering capacity of tissue only slightly affects the slope (fig. 3). Therefore, for small $\lambda$ values, two important contributions to the slope remain: one is related to continued gas exchange and the other to intra-acinar parallel concentration inhomogeneities (Luijendijk et al., 1980; de Vries et al., 1981; Paiva and Engel, 1981). The latter contribution is represented by the results for the perfusion-interrupted model in fig. 2 and table 1 , whereas the positive contribution of continued gas exchange is represented by the difference betwcen the two curves in each panel of fig. 2 . This contribution is nearly $\lambda$-independent for $\lambda$ values less than about 4.0. This will be explained in the Appendix, using classical tools of transpulmonary gas exchange. Consequently, one may expect that, under breath-to-breath steady-state conditions of gas exchange, the well-known opposite slopes for $\mathrm{O}_{2}$ and $\mathrm{CO}_{2}$ will cancel out to a large extent and give rise to only a slight slope for $\mathrm{N}_{2}$. 


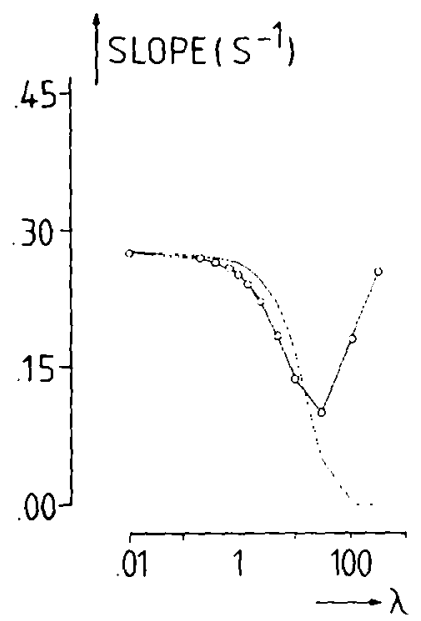

Fig. 3. The slope of the alveolar plateau plotted as a function of $;$ for the lung model 19:26-21:1. The comparison between the results for the lung models with and without lung tissue is shown for I) $==0.1$, where the solid curve represents the tissue-free model.

Finally, the calculations in the Appendix also show that the contribution of continued gas exchange to the slope is correlated with the alveolar ventilation, $\dot{\mathrm{V}} \mathrm{A}$. Therefore, a threefold increase in $\dot{V}_{A}$ in moving from condition I to condition III will proportionally increase the positive contribution of continued gas exchange to the slope for gases with a $\lambda$ value less than about 5.0 ( $\mathrm{sec}$ table 1).

THE SLOPE OF THE AI.VIOOLAR PI.ATYAU AS A FUNCTION OF THF MOLFCULAR DIFILLSION COIFFICIENT (I)

The influence of diffusion limitation in the gas phase on the slope of the alveolar plateau is calculated in an asymmetric lung model coded $19 ; 26-21 ; 1$ with different values of $D$. The choice of $N=19$ is based on the fact that the results of washout simulations with $\mathrm{N}=19$ in a previous investigation (Luijendijk $e$ ' al., 1980) approximated the experimental results better than with other generation numbers, like $N=16$ or $N=20$. Table 1 also shows that the steepest slopes are obtained for $N=19$ with $\mathrm{D}=0.22$ and $\hat{i}<c a .5 .0$. This indicates a specific interaction between $\mathrm{N}$ and the location of the transition zone, which is, among others, D-dependent. This can be explained by the fact that the slopes are steepest when the generation of division, $\mathrm{N}$, coincides approximately with the midpoint of the transition zone (Luijendijk et al., 1980, de Vries et al., 1981). In that case, a convective-diffusive interaction between reduced and nonreduced lung units is most pronounced, resulting in a changing gas concentration during expiration in the generations downstream. If the transition zone is too proximally or too peripherally located with regard to the fixed value of $\mathrm{N}$, the convective diffusive interaction between the 

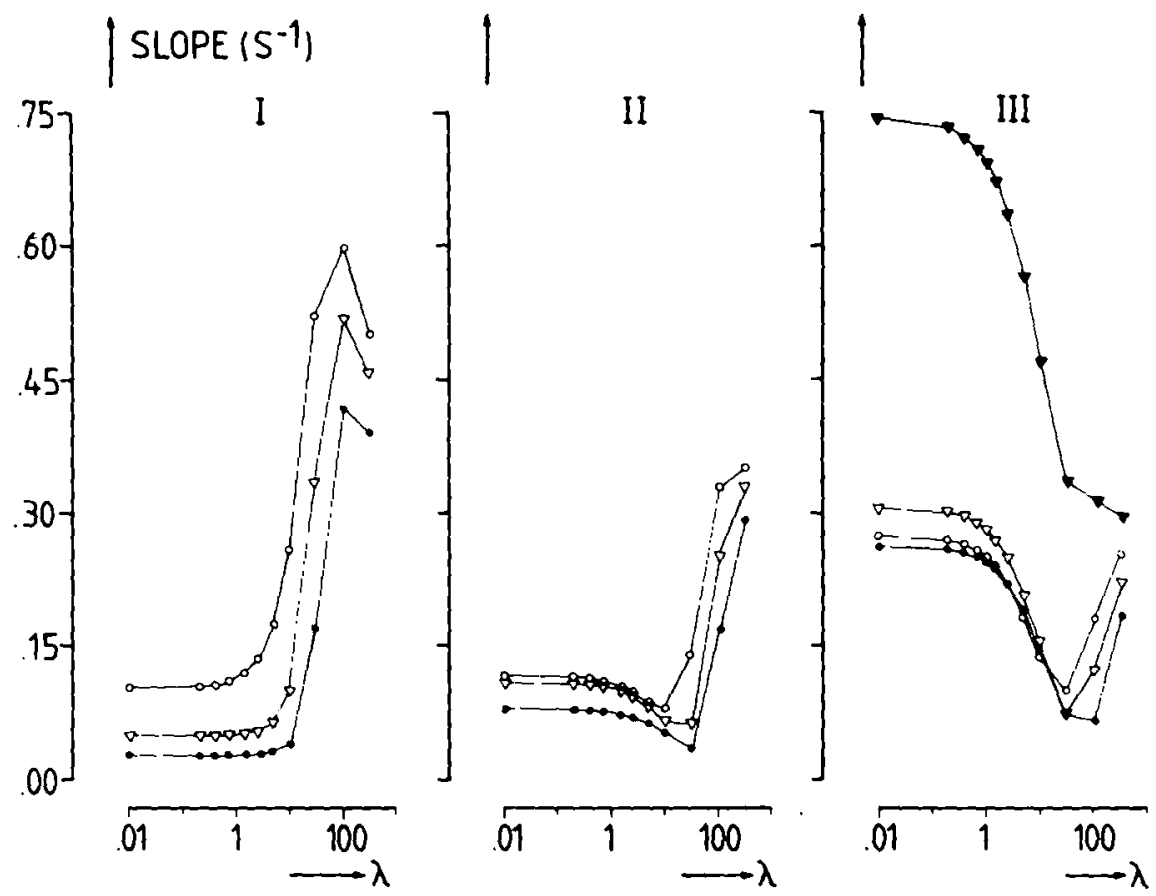

Fig. 4. The slope of the alveolar plateau plotted as a function of $\lambda$ for different D values. Sections I. II and III show the results for corresponding ventilation-perfusion conditions (see text), where open circles are used for $D=0.1$, triangles for $D=0.22$ and closed circles for $D=0.5$. In addition, section III shows the results under hyperbaric conditions (closed triangles. $\mathrm{D}=0,01$ )

unequal lung units can be neglected because cither the diffusion or the convection will overrule the other transport mechanism at the branch point. Therefore, a fixed choice of $\mathrm{N}$ may influence the qualitative relation between the results obtained with different values of $D$.

Figure 4 demonstrates the influence of $\mathrm{D}$ and exercise on the slope as a function of $\lambda$. In general, there is a stecper slope for heavy gases at a given $\lambda$ value, with one exception in condition $I I I$ for gases with a $\lambda$ value less than about 10.0 , where an inversion takes place for $D=0.1$ and $D=0.22$. This anomaly has to be ascribed to the specific interaction between $N$ and the location of the transition zone, as discussed above. Simulations under hyperbaric conditions $(D=0.01)$ show that, for ventilation -perfusion condition III, the calculated slopes are always steeper than for $\mathrm{D}=0.22$ (see fig. 4 .. III), supporting the general trend. These results obtained during washout of tracer gases supplied by the mixed venous blood are qualitatively similar to those for washout experiments from the alveolar space for light and heavy gases (Okubo and Piiper. 1974; Smidt, 1976; von Nieding et al., 1977).

It was shown in the previous section that, for gases with a $\lambda$ value less than about 10.0 , the positive contribution of continued gas exchange to the slope 
decreases in going from exercise to rest (fig 2). For tracer gases with identical $)$ values but different diffusive properties, we may expect that the relative contribution of diffusion limitation in the gas phase to the slope will be most pronounced when the contribution of continued gas exchange is least important, i.e., at rest. Consequently, the relative differences between the slopes as a function of $D$ will decrease with increasing excrcise (see fig. 4, panels I. II and III).

The simulation under hyperbaric conditions for gases with $\lambda<10.0$ results in relatively very steep slopes due to an extreme degree of stratification established during inspiration. This is clearly shown in fig. 5 where, for a gas of intermediate solubility, the end-inspiratory concentration profiles corresponding to normobaric and hyperbaric conditions are compared. Under hyperbaric conditions, at the onset of expiration there is an extreme degree of stratilication in the generations 18-21. This deep penetration of the transition zone into the acinus will not only delay the onset of phase II of the expiratory concentration curve but will also increase the slope of the alveolar plateau.

For gases with $\lambda$ values greater than about 10.0 , the continued gas exchange opposes the effects of stratification on the slope. This also explains why for highly soluble gases the slopes tend to converge, which is clearly shown in fig. 4 - III.

What is the significance of these results obtained by comparing the washout from pulmonary blood with that from alveolar space? Suppose that a healthy human subject at rest inhales low concentrations of $\mathrm{He}$ and $\mathrm{SF}_{6}$ in air until a complete equilibrium of gas concentration in the body is attained $\left(\mathrm{PI}=\mathrm{PA}_{\bar{A}}^{-}=\overline{\mathrm{P}} \overline{\mathrm{V}}\right.$, where $\mathrm{PI}$ is the inspired partial pressure, $\mathrm{PA}^{-}$is the mean alveolar partial pressure and $\mathrm{P}_{\mathrm{v}}$ is the partial pressure in mixed venous blood). According to previous calculations on washout from the alveolar space (de Vries et al., 1981) and the present model calculations, we are able to predict the behaviour of the slope during the subsequent multiple breath washout. In the first part of washout, the expired tracer gases
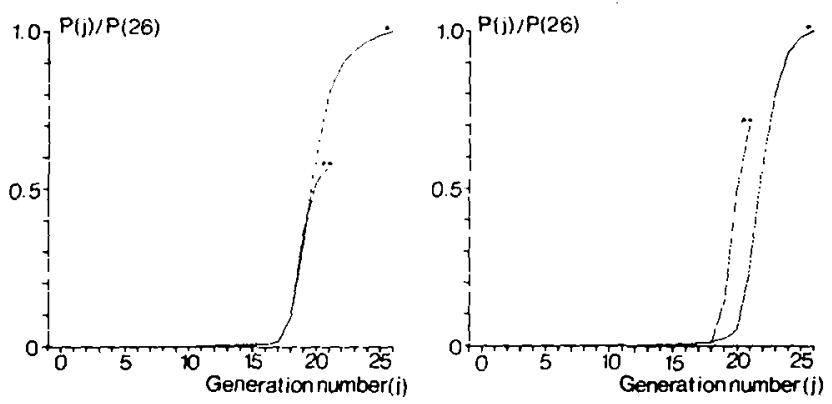

Iig. 5. The normalized partial pressure profile of a tracer gas with $i=0.7$ at the midway point of each generation versus generation number $(j)$ at the onset of expiration. The curves are related to ventilationperfusion condition 111 with normobaric $(D=0.1)$ and hyperbaric $(D=0.01)$ conditions on the left and right hand sides. respectively. The lung model $19: 26-21 ; \mathrm{I}$ is used and the asterisks * and ${ }^{* *}$ refer to the nonreduced and reduced parallel lung units, respectively. 
originate mainly from the alveolar space. Therefore, we predict that, for an initial small number of breaths, the expiratory concentration curves for both tracer gases will show a slightly increasing normalized slope, where the normalized slope for $\mathrm{SF}_{6}$ will remain significantly steeper than for He (de Vries et al., 1981). As the washout proceeds, the relative difference between the mixed venous and alveolar partial pressures of tracer gases will increase because the washout time constant for most body compartments is considerably larger than that for the alveolar space. Therefore, the positive contribution of continued gas exchange to the slope becomes more and more important. Consequently, in a later phase of washout, the steepness of the normalized slope for each gas will exceed the value in early washout and again the normalized slope of $\mathrm{SF}_{6}$ will remain steeper than for Hc. However, especially under strenuous excrcise conditions, the relative difference between these final slopes will decrease due to the dominant contribution of continued gas exchange.

\section{Conclusions}

This model study shows that the slope of the alveolar plateau during washout from mixed venous blood depends on the asymmetry of the intra-acinar branching pattern, the molecular diffusion coefficient of the tracer gas, the continued gas exchange and the buffering capacity of parenchymal tissue.

The relative contribution of diffusion limitation in the gas phase to the slope is for poorly soluble gases more pronounced at rest than under exercise conditions.

The normalized slopes for poorly and moderately soluble gases are only minimally dependent on $\lambda$.

In comparison with washout from alveolar space, the normalized slopes are less diffusion-dependent due to the contribution of continued gas exchange.

The slope of the alveolar plateau no longer needs to be explained by classical models consisting of parallel compartments with $\dot{V} / \dot{Q}$ inequality and synchronous ventilation.

\section{Appendix}

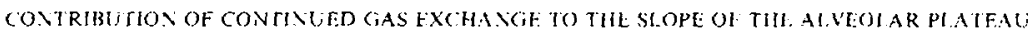

In the classical lung model with a nonoscillatory ventilation and perfusion, the ratio of the alveolar concentration of tracer gas, $C_{A}$, to the mixed venous concentration, $C \bar{v}$, can be described in a steady-state condition by (Wagner ef al., 1974):

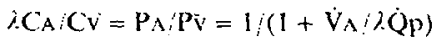

where $P A$ is the alveolar partial pressure, $P \bar{v}$ is the mixed venous partial pressure. $V_{A}$ is the alveolar ventilation and $\dot{Q} p$ the lung perfusion per minute. This equation can also be applied to compute approximately the mean end-tidal alveolar partial pressure in an oscillatory ventilated lung model. 
The mass transport across the alveolo-capillary membrane during expiration follows from:

$$
\mathrm{n}=\dot{i} \mathbf{Q} \cdot(C \bar{v}: \dot{C A}) \cdot \mathbf{t}
$$

where $t r$ is the expiration time.

Substitution of eq. 3 in eq. 4 yields for the relative increase in alvcolar partial pressure during expiration:

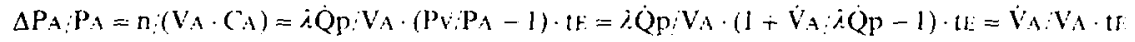

where $V_{A}$ is the alveolar volume

Hence, the relative increase in alveolar partial pressure during cxpiration is $\lambda$-independent, but $\checkmark$-dependent. In fact. the alveolar partial pressure increases nearly exponentially during expiration. Therefore, a linear approximation of this inerease is allowed only when $\triangle P A$ is considerably smaller than $\left(\mathrm{P}_{\bar{V}}-\mathrm{P}_{\mathrm{A}}\right)$. e.g.. $\Delta \mathrm{P}_{\mathrm{A}}:\left(\mathrm{P} \mathrm{V}-\mathrm{P}_{\mathrm{A}}\right)<0.3$.

Application of eqs. 3 and 5 yields:

$$
\Delta \mathrm{P}_{A_{i}}\left(\mathrm{P}_{\overline{\mathrm{V}}}-\mathrm{P}_{\mathrm{A}}\right)=\left(\left(\dot{V}_{\mathrm{A}} \cdot \mathrm{P}_{\mathrm{A}} \cdot \mathrm{tr}_{\mathrm{H}}\right): \mathrm{V}_{\mathrm{A}}\right) \cdot i \dot{\mathrm{Q}}_{\mathrm{p}}\left(\dot{\mathrm{V}}_{\mathrm{A}} \cdot \mathrm{P}_{\mathrm{A}}\right)=i \dot{Q} \mathrm{P}_{\mathrm{P}} \cdot \mathrm{tr}: \mathrm{V}_{\mathrm{A}}<0.3
$$

For condition 1 , where $Q p=7.5 \mathrm{~L} . \mathrm{min}, \mathrm{tE}=2.0 \mathrm{sec}$ and $\mathrm{V}_{\mathrm{A}}=3.25 \mathrm{~L}: \mathrm{A} \rightarrow\langle 3.9$;

For condition 11 , where $\mathrm{Qp}=10.0 \mathrm{~L} \mathrm{~min}, \mathrm{t} t=1.5 \mathrm{sec}$ and $\mathrm{V}_{\mathrm{A}}=3.25 \mathrm{~L}: \rightarrow i<3.9$;

For condition III, where $\dot{Q p}=20.0 \mathrm{~L}: \mathrm{min}, \mathrm{tI}:=1.0 \mathrm{sec}$ and $V_{\mathrm{A}}=3.25 \mathrm{~L}: \rightarrow i<3.0$.

In conclusion: for $i<$ about 4.0 , the contribution of continued gas exchange to the slope of the alveolar plateau will only slightly depend on the $j$. value.

\section{Acknowledgements}

The authors express their thanks to Mr. J. J. H. de Wit for his technical assistance and to Mrs. S. B.P.A. Vesters for her assistance with the manuscript.

\section{References}

Cander. L. (1959). Solubility of inert gases in human lung tissuc. J. Appl. Physiol. 14:538.540.

Chang, H., R. T. Cheng and I. E. Farhi (1973). A model study of gas diffusion in alveolar sacs. Respir. Physiol. 18: 386-397.

Davidson. M. R. and L. A. Engel (1982). (Gas transport in an asymnetrical acinus. Bull. Fur. Physiopathol. Respir. 18: 203-214.

Hansen. J. 1., 1.. P. Ampaya. G. H. Bryant and J. J. Navin (1975). Branching pattern of airways and air spaces of a single human terminal bronchiole. J. Appl. Physiol. 38: $983-989$

Hansen. J. E and E. P. Ampaya (1975). Human air space shapes sizes, areas and volumes. $J . A p p l$. Physiol. 38: 990995.

Lacquet. L. M. (1976). Convection and diffusion in the airways. Thesis. Catholic Lniversity, I.euven. Bclgium.

Luijendijk. S.C.M. A. Zwart, W. R. de Vries and W. M. Salet (1980). The sloping alvcolar platicau at synchronous ventilation. Pfügers Arch. 384: 267-277.

Mon, F. and J.S. L'ttman (1976). Monte Carlo simulation of simultaneous gas flow and diffusion in an asymmetric distal puimonary airway model. Bull. Wath. Biol. 38: 161 192.

Vieding, G. von, H. I.̈llgen, U. Smidt and H. Linde (1977). Simultaneous washout of helium and suiphur hexafluoride in healthy subjects and patients with chronic bronchitis, bronchial asthma and emphysema. An. Re'v. Respir. Dis. 116: $649-660$. 
Okubo, T. and J. Piiper (1974). Intrapulmonary gas mixing in excised dog lung lobes studied by simultaneous washout of two inert gases. Respir. Physiol. $21: 223.239$.

Paiva, M. and L. A. Fngel (1979). Pulmonary interdependence of gas transport. J. Appl. Physiol. 47: 296-305.

Paiva, M. and 1. A. Engel (1981). The anatomical basis for the sloping $N_{2}$ plateau. Respir. Physiol. 44: $325 \cdot 337$.

Robertson. H.T. and M.P. Hastala (1977). Nlevated alveolar $P_{(\mathrm{C})}$. relative to predicted values during normal gas exchange. J. Appl. Physiol. 43: 357-364.

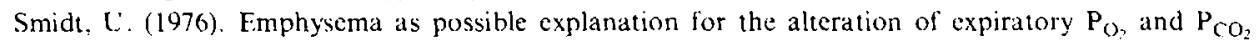
curves. Bull. Lur. Physiopathol. Respir. 12: 605-624.

Ulmer, W.T., G. Reichel and D. Nolte (1976). In: Dic Lungenfunktion. Stuttgart. Georg-ThiemeVerlag, p. 142.

Lltman, J.S. and M.W. Thomas (1979). Longitudinal mixing in pulmonary airways: comparison of inspiration and expiration. J. Appl. Physiol. 46: $799-805$.

Vries, W.R. de, S.C.M. Luijendijk and A. Zwart (1981). Helium and sulfur hexafluoride washout in asymmetric lung models. J. Appl. Physiol. $51: 1122 \cdot 1130$.

Vries, W. R. de. and S. C. M. L.uijendijk (1982). Shape of single-breath washout curves of gases with different diffusion coefficients and blood solubilitics. Bull. Eur. Physiopathol. Respir. 18: 215227.

Wagner, P.D., H.A. Saltzman and J.B. West (1974). Measurements of continuous distributions of ventilation-perfusion ratios: theory. J. Appl. Physiol. 36: 588-599.

Wagner, P. D., P.F. Naumann and R. B. Laravuso (1974). Simuhtaneous measurement of eight foreign gases in blood by gas chromatography. J. Appl. Physiol. 36: 600-605.

Weibel. E. R. (1963). In: Morphometry of the Human Lung. Berlin. Springer-Verlag. p. 109.

Z.wart, A. S. C. M. Luijendijk and W. R. de Vries (1982). Simulated pulmonary gas exchange dependent on the blood solubility and diffusion coefficient. (Submitted.) 